\title{
Risk Factors for Disease Progression and Prognosis Analysis in Pediatric Head and Neck Rhabdomyosarcoma Patients: a Historical Cohort Study From a Single Center
}

\section{Yuan Wen}

Beijing Tongren Hospital, Capital Medical University

Weiling Zhang

Beijing Tongren Hospital, Capital Medical University

Yi Zhang

Beijing Tongren Hospital, Capital Medical University

Huimin $\mathrm{Hu}$

Beijing Tongren Hospital, Capital Medical University

Jing Li

Beijing Tongren Hospital, Capital Medical University

Dongsheng Huang ( $\sim$ huangdongshengtr@163.com )

Beijing Tongren Hospital, Capital Medical University https://orcid.org/0000-0002-0987-2273

Research article

Keywords: rhabdomyosarcoma, pediatric, disease progression, prognosis, risk factor, head and neck

Posted Date: August 3rd, 2020

DOI: https://doi.org/10.21203/rs.3.rs-51132/v1

License: (c) (i) This work is licensed under a Creative Commons Attribution 4.0 International License.

Read Full License 


\section{Abstract}

Background: The purposes of this study are to explore risk factors for disease progression during primary treatment, and to analyze disease prognosis of different event groups in a historical cohort of 56 pediatric patients with head and neck rhabdomyosarcoma.

Methods: From June 1st, 2016 to June 30th, 2019, a historical cohort of 56 pediatric patients diagnosed as head and neck rhabdomyosarcoma was chosen. Clinical data were collected including general information about diagnosis, treatment, prognostic factors, and follow up results. Patients were divided into non-event group, disease-progression group and disease-relapse group according different types of events. Comparison among the three groups was made to reveal different clinical features and prognosis. Patients were further divided into disease-progression group and non-progression group to explore risk factors for disease progression, with univariate and multivariate analysis.

Results: The median follow-up time of all 56 patients was 31.8 months (range 3.5-74.6 months). There were 30 non-event patients, 12 disease relapse patients, and 14 disease-progression patients. The estimated 5-year overall survival rates were $100.0 \%, 82.5 \%$, and $11.9 \%$ for non-event, disease-relapse and disease-progression patients respectively $(p<0.001)$. Compared with non-event patients, diseaseprogression patients had higher rates of tumor size $>5 \mathrm{~cm}(p=0.008)$ and non-initial radiation therapy $(p=0.001)$. Compared with disease-relapse patients, disease-progression patients had a higher rate of tumor size $>5 \mathrm{~cm}(\mathrm{p}=0.002)$, and higher ranks of surgical-pathologic group $(p=0.009)$ and risk group $(p<0.001)$. Compare with non-progression patients, univariate analysis demonstrated that tumor size $>5 \mathrm{~cm}$, alveolar histology, non-initial radiation therapy, and higher ranks of surgical-pathologic group as well as risk group were statistically significant risk factors for disease progression. The multivariate analysis result showed tumor size $>5 \mathrm{~cm}(\mathrm{OR}=6.75,95 \% \mathrm{Cls} 1.02-44.78)$, alveolar histology (OR=7.10, $95 \% \mathrm{Cls} 1.08-46.57)$, and non-initial radiation therapy (OR=15.26, $95 \% \mathrm{Cls} 2.38-97.70)$ to be independent risk factors for disease progression.

Conclusions: Pediatric head and neck rhabdomyosarcoma patients with disease progression have significantly worse overall survival, and the prognosis is obviously different from patients with disease relapse and patients without events. Tumor size $>5 \mathrm{~cm}$, alveolar histology and non-initial radiation therapy are independent risk factors for disease progression. Patients with disease progression tend to have higher ranks of surgical-pathologic group and risk group.

\section{Background}

Rhabdomyosarcoma (RMS) is a malignant tumor that can develop in any part of the body, with a primitive mesenchymal cell origin and a propensity for striated muscle differentiation [1-3]. It accounts for over $50 \%$ of childhood soft tissue sarcoma $[4,5]$. The estimated incidence is about 4.5 cases per 1 million children and young adults aged under 20 years [2,3]. Over the last 3 decades great survival improvement has been achieved in RMS patients with 5-year overall survival (OS) exceeding $70 \%[2,5]$. And this is 
mainly attributed to risk stratification-based treatment plan and multimodal treatment strategy [5]. But the survival of patients with relapsed and advanced disease remains poor with 5 -year OS about $30 \%[2,5,6]$.

Head and neck rhabdomyosarcoma (HNRMS) comprises $40 \%$ of all RMS and is the most common region of primary tumor sites $[7,8]$. The primary sites of HNRMS include orbit, parameningeal and nonparameningeal nonorbit head and neck. About $50 \%$ of HNRMS cases have unfavorable primary sites (parameningeal region), arising in the middle ear/mastoid, nasopharynx/nasal cavity, paranasal sinus, parapharyngeal region, or pterygopalatine/infratemporal fossa $[7,8]$. Treatment for HNRMS can be a significant challenge due to the likelihood of infeasible radical surgery, considering the complicated anatomy and functional/cosmetic sequelae [8].

Survival related events of RMS include disease progression, disease relapse, second tumor and death of any cause [9-11]. In the vast majority of studies, the prognosis of patients with events is analyzed as a single group and event free survival (EFS) is constantly used, which is actually a highly effective index for prognosis [9-11]. However, we noticed the heterogeneity of these patients with events, that those with disease progression had the most frustrating outcome compared with others. Yet patients with disease progression have seldomly been analyzed as a separate group [12]. Base on the above we tried to explore the risk factors for disease progression, and to compare the prognosis of patients with disease progression with others in a historical cohort of pediatric HNRMS patients.

\section{Methods}

\section{Study design}

This is a historical observational cohort study, including all HNRMS patients diagnosed and treated in our pediatric department from June 1st, 2013 to June 30th, 2019.

\section{Diagnostic procedure, risk stratification and treatment protocol}

The uniform diagnostic procedure, risk stratification and treatment protocol for this cohort have been described in details in our previously report [13]. Generally, site, size, primary tumor invasiveness, nodal involvement and metastatic status were determined by pretreatment imaging and test, and patient's stage was assigned according to the pretreatment staging system of Soft Tissue Sarcoma Committee of Children's Oncology Group [14]. Primary surgical resection was attempted or only a biopsy was done in case of infeasible complete resection, and group was assigned according to IRS surgical-pathologic group system [14]. At last the risk group was assigned with comprehensive consideration of stage, group, and pathologic subtype results, according to the risk group classification of Soft Tissue Sarcoma Committee of Children's Oncology Group [14].

Chemotherapy was guided by risk group with reference to COG D9602 subgroup B VAC regimen (vincristine, dactinomycin, cyclophosphamide) for low risk group [15], and COG D9803 standard VAC regimen for intermediate risk group [16]. For high risk group the chemo plan was based on standard VAC 
regimen with an optional combination of anthracyclines, platinum drugs, etoposide or irinotecan. Radiation therapy (RT) was recommended to all patients except for Group \& embryonal patients, and RT dose was determined according to the recommendation of Soft Tissue Sarcoma Committee of Children's Oncology Group. As previously stated [13], that in $40 \%$ (22/56) of our pediatric HNRMS patients RT was not included as a component of the initial treatment plan, which was mainly attributed to parental refusal due to concerns about long-term RT toxicity or blind confidence in chemotherapy alone.

\section{Follow up and data collection}

All patients were closely followed up since diagnosis, and all the clinical data regarding diagnosis, risk stratification, treatment and outcome were collected.

OS was defined as survival from diagnosis to death of any cause. Disease progression was defined as primary tumor enlargement, and/or new lesions, and/or metastasis during primary treatment course. Disease relapse was defined as recurrence of RMS in any form after last treatment during off therapy surveillance. Survival related events including disease progression, disease relapse, second tumor and death of any cause, and only the first progression and relapse were discussed and analyzed.

\section{Grouping and statistical methods}

According to survival related events and the occurrence of disease progression during primary treatment, all patients were divided into Group PD (patients with disease progression) and Group NPD (patients without disease progression). Group NPD was further divided into Group NE (patients with none event) and Group RD (patients with disease relapse).

OS as time-to-event distribution was estimated with Kaplan-Meier method. Survival differences between groups were compared with log rank tests. Clinical data of Group NE, Group RD and Group PD were compared with each other to reveal differences. Dichotomous categorical variables were compared using chi-squared test or Fisher's exact test between groups, while ordinal categorical variables (stage, surgicalpathologic group and risk group) were compared using Kruskal-Wallis $\mathrm{H}$ test. Univariate analysis between Group NPD and Group PD was performed using chi-squared test/Fisher's exact test (for dichotomous categorical variables) and Cochran-Armitage trend test (for ordinal categorical variables). Multivariate analysis was performed using binary logistic regression model to explore risk factors for disease progression and adjust confounding factors. Odds ratios (OR) with $95 \%$ confidence intervals (Cls) were calculated. Bonferroni adjustment was used to control type I error if more than two groups were compared. A p value $<0.05$ was considered statistically significant. Data were analyzed with IBM SPSS Statistics 26.0.

\section{Results}

\section{General information}


The cohort consisted of 56 pediatric HNRMS patients, who were admitted into our pediatric department from June $1 \mathrm{st}, 2013$ to June 30th, 2019. The diagnoses were confirmed by pathology results. Male to female ratio was 31:25, and the follow up time of different groups was showed in Table1. One patient was lost to follow up at 3.5 months after diagnosis due to moving abroad, who belongs Group NPD/Group NE.

\section{Survival results}

The estimated 5-year OS of all patients was $69.9 \%$. The estimated 5-year OS of Group NE, Group RD, and Group PD were $100.0 \%, 82.5 \%$, and $11.9 \%$ respectively, and log rank test showed a statistically significant difference $(p<0.001)$. (Fig 1A) Further pairwise comparisons between the three groups were done with Bonferroni adjustment ( $p<0.0167$ was considered statistically significant). The results showed statistically survival differences between Group NE and Group PD ( $<<0.001)$, as well as Group RD and Group PD ( $p=0.001)$, but no difference between Group NE and Group RD ( $p=0.043)$. The estimated 5-year OS of Group NPD and Group PD were $94.1 \%$ and $11.9 \%$, with a statistically significant difference tested by log rank test $(\mathrm{p}<0.001)$. (Fig 1B)

\section{Clinical characteristic comparisons between Group NE, Group RD, and Group PD}

The detailed clinical characteristics of Group NE, Group PD and Group RD were showed in Table 2. Comparisons were made between these groups, and when an overall comparison achieved statistical significance, pairwise comparisons were performed with Bonferroni adjustments.

According to the results, compared with Group NE, patients in Group PD had higher rates of tumor size $>5 \mathrm{~cm}(p=0.008)$ and non-initial radiation therapy $(p=0.001)$. Compared with Group RD, patients in Group PD had a higher rate of tumor size $>5 \mathrm{~cm}(p=0.002)$, and higher ranks of surgical-pathologic group $(p=0.009)$ and risk group $(p<0.001)$.

\section{Univariate analysis of risk factors for disease progression}

Considering the significantly lower OS of Group PD and referring to previously reported risk factors for survival, we performed univariate comparison of risk factors between Group NPD and Group PD. The results are showed in Table 3.

The univariate analysis results showed that tumor size $>5 \mathrm{~cm}$, alveolar histology, non-initial RT, and higher ranks of surgical-pathologic group and risk group were statistically significant risk factors for disease progression.

\section{Multivariate analysis of risk factors for disease progression}

Binary logistic regression model was used for multivariate analysis, and all statistically significant variables in univariate analysis were included in the model in order to adjust confounding factors, including tumor size, histologic subtype, surgical-pathologic group, risk group and initial RT. Before using 
binary logistic regression model, a collinearity diagnostic test was performed, which revealed the collinearity of variable surgical-pathologic group and variable risk group (condition index 20.6, variance proportion of surgical-pathologic group 0.78 and risk group 0.63). Since risk group is a comprehensive variable containing information that overlaps with the rest variables, it was excluded from the logistic model. Variable surgical-pathologic group contained unique information about surgical results, and a repeated collinearity diagnostic test showed satisfactory results after excluding variable risk group (condition index 15.6 with no variance proportion exceeding 0.5 ), so it was included in the final logistic model. The multivariate analysis results are showed in Table 4, and the final logistic model was statistically significant (Chi-square value 27.1, $p<0.001$ ). According to the results, tumor size $>5 \mathrm{~cm}$ $(\mathrm{OR}=6.75,95 \% \mathrm{Cls} 1.02-44.78)$, alveolar histology (OR=7.10, 95\%Cls 1.08-46.57), and non-initial RT $(\mathrm{OR}=15.26,95 \% \mathrm{Cls} 2.38-97.70)$ were confirmed as independent risk factors for disease progression. While higher ranks of surgical-pathologic group was not an independent risk factor.

\section{Discussion}

The prognosis of RMS has been greatly improved over the last 3 decades, thanks to the series of clinical trials carried out by the COG Soft Tissue Sarcoma Committee in North America, the European pediatrics Soft Tissue Sarcoma Study Group (EpSSG), etc. [2, 5]. Although 5-year OS now exceeds $70 \%$, survival of patients with recurrent and advanced disease is still poor, with 5-year OS about $30 \%[2,5,6]$. Survival related events include disease progression, disease relapse, second tumor and death of any cause [9-11]. Treatment related second tumor and death of other cause (tumor progression or relapse unrelated cause) are rare, while disease progression and relapse comprise almost all events $[9,17,18]$. In most studies patients with disease progression and relapse were analyzed as a combined single group, using EFS rate to evaluate prognosis. The estimated EFS rate should be lower than OS rate, which is due to the fact that some patients with disease progression or relapse could still be salvaged with intensive/second-line treatment. Compared with OS, EFS is a more conservative estimation yet actually effective.

Generally, disease progression represents early treatment failure [12], which includes primary tumor enlargement, and/or new lesions, and/or metastasis during primary treatment course. Patients with disease progression respond poorly to the initial treatment, and primary tumor enlargement, local invasion, regional nodal involvement, and even metastasis can be anticipated. Disease relapse represents late treatment failure $[19,20]$, which means recurrence of RMS in any form after last treatment during off therapy surveillance. Patients with disease relapse usually respond well to the initial treatment and end with complete remission. Later the tumor recurs from incompletely eradicated micro residual of primary site, regional nodes, or metastatic foci.

The biological processes of disease progression and relapse are distinct, leading to distinct prognosis. Our survival results confirmed the heterogeneity of this group of patients with events, that Group PD had significantly worse survival expectation compared with Group RD. Clinical features of Group PD were not only different from Group NE, but also Group RD. Interestingly clinical features of Group NE and Group RD were more alike. Pairwise comparisons revealed that Group PD tended to have larger tumor size, higher 
ranks of surgical pathologic group (unresectability) and risk group (advanced disease status), which may indicate a more malignant biological behavior.

Clinically disease progression indicates resistance to current treatment, and once progression confirmed the treatment is usually enhanced or switched to second-line regimen. But disease relapse is more likely attributed to insufficient treatment to a responsive tumor, which could be inadequate local control (surgery and/or RT), and/or inadequate systemic control (intensity and/or duration of chemotherapy). A multicenter open-label randomized phase 3 trial finished by EpssG recently showed that maintenance treatment with vinorelbine and low-dose oral cyclophosphamide for patients with high-risk rhabdomyosarcoma in complete remission after standard treatment improves OS [3]. This survival improvement was the result of the lower recurrence rate. The authors explained that the improvement of OS might be attributed to prolonged chemotherapy or the combination of vinorelbine and cyclophosphamide might have killed the small amount of residual disease remaining at the end of standard treatment.

A lot of prognostic factors for survival have been reported. Considering the poor survival of Group PD, we explored these factors for disease progression. The univariate and multivariate analysis results confirmed tumor size, histology subtype and initial RT as independent prognostic factors for disease progression. Although surgical-pathologic group showed statistical significance on univariate analysis, it was not an independent prognostic factor for disease progression on multivariate analysis. This is not difficult to understand that the resectability of the primary tumor is affected by many factors, such as anatomy (site/tumor size), invasiveness (regional or distal involvement/histology subtype), etc., which makes surgical pathologic group unlikely an independent prognostic factor. Our results of the Cochran-Armitage test for trend indicated that the possibility of disease progression increased with the ranks of surgical pathologic group and risk group, which meant patients with unresectable tumor and advanced disease were more likely to develop disease progression.

We previously reported that the patterns of disease progression and relapse of this cohort were mainly local, which was consistent with other published reports [9, 21]. A cure for RMS depends firstly on local control (eradication of the primary tumor by surgery and/or RT), then on systemic control (eradication of the micro and metastatic residual) [5]. Local tumor is the main burden of the whole disease, including the primary site and regional nodes. Local eradication is more responsible for complete remission/cure of the disease, and failed local control is also more responsible for disease progression and relapse. This explains why the main pattern is local.

The importance of local control by surgery and/or RT has widely been reported and confirmed in this cohort $[8,13,22]$. We reported that non-initial RT impairs OS and EFS by causing local failure in this cohort. And it has been reported that delayed RT may cause treatment failure in parameningeal RMS patients [23, 24], also delayed RT may cause early treatment failure (mainly disease progression) in intermediate risk RMS patients [9]. This explains why initial RT was an independent prognostic factor for disease progression. 
Tumor size $>5 \mathrm{~cm}$ has been widely reported as a risk factor for survival especially in refractory patients (patients with disease progression) and recurrent patients (patients with disease relapse) [25-28], and is the strongest predictor of local failure [29]. It is widely reported RMS patients with alveolar histology have worse survival than embryonal type, especially those alveolar patients with PAX-FOXO1 fusion gene [5, $10,11]$. The biological behavior is very different between the alveolar and embryonal histologic subtypes, with alveolar (especially PAX-FOXO1 positive) subtype more aggressive and easier to infiltrate and metastasize $[5,30]$.

From the above, we can explain these risk factors from two angles. Firstly, failed local control is prone to disease progression. If the tumor has one or more of the following features, which include large (tumor size $>5 \mathrm{~cm}$ ), aggressive (alveolar type), unresectable (high surgical pathologic rank) and not receiving RT initially (non-initial RT), the local control is impossible by chemotherapy alone, which would cause disease progression. Secondly, advanced disease is prone to disease progression. If the tumor has progressed into an advance status, especially with metastasis (high rank of risk group), with infeasible surgery (high rank of surgical-pathologic group), chemotherapy would appear feeble and futile in front of such large tumor burden.

\section{Limitations}

Unfortunately, we could not acquire nearly half of the PAX-FOXO1 gene test results of our alveolar patient in this cohort, and we didn't make any statistical analysis about it. Positive fusion gene status is certainly a very important risk factor for survival and predicts more malignant tumor biological behavior $[5,10,11$, 30], which is assumed to be a risk factor for disease progression. The reported positive rate of PAXFOX01 fusion gene is about $80 \%$ in alveolar RMS $[5,10]$, making our result of alveolar histologic subtype being a risk factor for disease progression a more conservative one. The fact that we did not identify surgical-pathologic Group $\otimes$ patients implied a selection bias, and this is probably because we are a tertial center with domestically high-ranking ophthalmology and otorhinolaryngology head \& neck surgery department. All these could limit the generalizability of this study.

\section{Conclusions}

In conclusion, pediatric head and neck rhabdomyosarcoma patients with disease progression have significantly worse overall survival, and the prognosis is obviously different from patients with disease relapse and patients without events. Tumor size $>5 \mathrm{~cm}$, alveolar histology and non-initial RT are independent risk factors for disease progression. Patients with disease progression tend to have higher ranks of surgical-pathologic group and risk group.

\section{List Of Abbreviations}

RMS: Rhabdomyosarcoma 
OS: Overall Survival

HNRMS: Head and Neck Rhabdomyosarcoma

EFS: Event Free Survival

VAC: Vincristine, Dactinomycin, Cyclophosphamide.

RT: Radiation Therapy

Group PD: Patients with Disease Progression Group

Group NPD: Patients with None Disease Progression Group

Group NE: Patients with None Event Group

Group RD: Patients with Disease Relapse Group

OR: Odds Ratio

Cl: Confidence interval

COG: Children's Oncology Group

IRS: Intergroup Rhabdomyosarcoma Study

EpSSG: European pediatrics Soft Tissue Sarcoma Study Group

\section{Declarations}

\section{Ethics approval and consent to participate}

This study was conducted in accordance with the declaration of Helsinki and with approval from the Ethics Committee of Capital Medical University, Beijing Tongren Hospital. Written informed consent was obtained from all participants' guardians.

\section{Consent for publication}

Not applicable.

\section{Availability of data and materials}

Not applicable.

\section{Competing interests}

The authors declare that they have no competing interests. 


\section{Funding}

This study was funded by the Special Fund of the Pediatric Medical Coordinated Development Center of Beijing Hospitals Authority (No. XTZD20180203), and Beijing Hospitals Authority Mission Plan (Code: DFL20180201)

\section{Authors' Contubutions}

YW designed the study, collected, analyzed and interpreted all the data, and completed the manuscript writing. WZ, YZ, HH, JL contributed in clinical data accumulation and collection, and participated in study design. All authors read and approved the final manuscript. DH initiated the study and participated in designing, did the critical revision of the manuscript, and provided the funding.

\section{Acknowledgements}

Not applicable.

\section{References}

1. Bisogno G, Jenney M, Bergeron C, Gallego Melcon S, Ferrari A, Oberlin O, Carli M, Stevens M, Kelsey A, De Paoli A et al: Addition of dose-intensified doxorubicin to standard chemotherapy for rhabdomyosarcoma (EpSSG RMS 2005): a multicentre, open-label, randomised controlled, phase 3 trial. Lancet Oncol 2018, 19(8):1061-1071.

2. Chen C, Dorado Garcia H, Scheer M, Henssen AG: Current and Future Treatment Strategies for Rhabdomyosarcoma. Front Oncol 2019, 9:1458.

3. Bisogno G, De Salvo GL, Bergeron C, Gallego Melcon S, Merks JH, Kelsey A, Martelli H, Minard-Colin V, Orbach D, Glosli $\mathrm{H}$ et al: Vinorelbine and continuous low-dose cyclophosphamide as maintenance chemotherapy in patients with high-risk habdomyosarcoma (RMS 2005): a multicentre, open-label, randomised, phase 3 trial. Lancet Oncol 2019, 20(11):1566-1575.

4. Amer KM, Thomson JE, Congiusta D, Dobitsch A, Chaudhry A, Li M, Chaudhry A, Bozzo A, Siracuse B, Aytekin MN et al: Epidemiology, Incidence, and Survival of Rhabdomyosarcoma Subtypes: SEER and ICES Database Analysis. J Orthop Res 2019, 37(10):2226-2230.

5. Skapek SX, Ferrari A, Gupta AA, Lupo PJ, Butler E, Shipley J, Barr FG, Hawkins DS:

Rhabdomyosarcoma. Nat Rev Dis Primers 2019, 5(1):1.

6. Yohe ME, Heske CM, Stewart E, Adamson PC, Ahmed N, Antonescu CR, Chen E, Collins N, Ehrlich A, Galindo RL et al: Insights into pediatric habdomyosarcoma research: Challenges and goals. Pediatr Blood Cancer 2019, 66(10):e27869.

7. Owosho AABCD, Huang SCM, Chen SM, Kashikar SD, Estilo CLD, Wolden SLM, Wexler LHM, Huryn JMD, Antonescu CRM: A clinicopathologic study of head and neck rhabdomyosarcomas showing FOX01 fusion-positive alveolar and MYOD1-mutant sclerosing are associated with unfavorable outcome. Oral Oncol 2016, 61:89-97. 
8. Defachelles AS, Rey A, Oberlin O, Spooner D, Stevens MC: Treatment of nonmetastatic cranial parameningeal thabdomyosarcoma in children younger than 3 years old: results from international society of pediatric oncology studies MMT 89 and 95. J Clin Oncol2009, 27(8):1310-1315.

9. Casey DL, Chi YY, Donaldson SS, Hawkins DS, Tian J, Arndt CA, Rodeberg DA, Routh JC, Lautz TB, Gupta AA et al: Increased local failure for patients with intermediate-risk rhabdomyosarcoma on ARST0531: A report from the Children's Oncology Group. Cancer 2019, 125(18):3242-3248.

10. Skapek SX, Anderson J, Barr FG, Bridge JA, Gastier-Foster JM, Parham DM, Rudzinski ER, Triche T, Hawkins DS: PAX-FOXO1 fusion status drives unfavorable outcome for children with rhabdomyosarcoma: a children's oncology group report. Pediatr Blood Cancer 2013, 60(9):14111417.

11. Hibbitts E, Chi YY, Hawkins DS, Barr FG, Bradley JA, Dasgupta R, Meyer WH, Rodeberg DA, Rudzinski ER, Spunt SL et al: Refinement of risk stratification for childhood rhabdomyosarcoma using FOXO1 fusion status in addition to established clinical outcome predictors: A report from the Children's Oncology Group. Cancer Med 2019, 8(14):6437-6448.

12. Minn AY, Lyden ER, Anderson JR, Million L, Arndt CA, Brown K, Hawkins DS, Donaldson SS: Early treatment failure in intermediate-risk rhabdomyosarcoma: results from IRS-IV and D9803-a report from the Children's Oncology Group. J Clin Oncol 2010, 28(27):4228-4232.

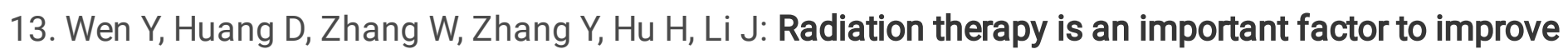
survival in pediatric patients with head and neck rhabdomyosarcoma by enhancing local control: a historical cohort study from a single center. BMC Pediatr 2020, 20(1):265.

14. Malempati S, Hawkins DS: Rhabdomyosarcoma: review of the Children's Oncology Group (COG) Soft-Tissue Sarcoma Committee experience and rationale for current COG studies. Pediatr Blood Cancer 2012, 59(1):5-10.

15. Raney RB, Walterhouse DO, Meza JL, Andrassy RJ, Breneman JC, Crist WM, Maurer HM, Meyer WH, Parham DM, Anderson JR: Results of the Intergroup Rhabdomyosarcoma Study Group D9602 protocol, using vincristine and dactinomycin with or without cyclophosphamide and radiation therapy, for newly diagnosed patients with low-risk embryonal habdomyosarcoma: a report from the Soft Tissue Sarcoma Committee of the Children's Oncology Group. J Clin Onco/2011, 29(10):13121318.

16. Arndt CA, Stoner JA, Hawkins DS, Rodeberg DA, Hayes-Jordan AA, Paidas CN, Parham DM, Teot LA, Wharam MD, Breneman JC et al: Vincristine, actinomycin, and cyclophosphamide compared with vincristine, actinomycin, and cyclophosphamide alternating with vincristine, topotecan, and cyclophosphamide for intermediate-risk rhabdomyosarcoma: children's oncology group study D9803. J Clin Oncol2009, 27(31):5182-5188.

17. Lautz TB, Chi YY, Tian J, Gupta AA, Wolden SL, Routh JC, Casey DL, Dasgupta R, Hawkins DS, Rodeberg DA: Relationship between tumor response at therapy completion and prognosis in patients with Group III rhabdomyosarcoma: A report from the Children's Oncology Group. Int J Cancer 2020. 
18. Setty BA, Stanek JR, Mascarenhas L, Miller A, Bagatell R, Okcu F, Nicholls L, Lysecki D, Gupta AA: VIncristine, irinotecan, and temozolomide in children and adolescents with relapsed rhabdomyosarcoma. Pediatr Blood Cancer 2018, 65(1).

19. Winter S, Fasola S, Brisse H, Mosseri V, Orbach D: Relapse after localized rhabdomyosarcoma: Evaluation of the efficacy of second-line chemotherapy. Pediatr Blood Cancer 2015, 62(11):19351941.

20. Dantonello TM, Int-Veen C, Schuck A, Seitz G, Leuschner I, Nathrath M, Schlegel PG, Kontny U, Behnisch W, Veit-Friedrich I et al: Survival following disease recurrence of primary localized alveolar rhabdomyosarcoma. Pediatr Blood Cancer 2013, 60(8):1267-1273.

21. Vaarwerk B, Mallebranche C, Affinita MC, van der Lee JH, Ferrari A, Chisholm JC, Defachelles AS, De Salvo GL, Corradini N, Minard-Colin V et al: Is surveillance imaging in pediatric patients treated for localized rhabdomyosarcoma useful? The European experience. Cancer 2020, 126(4):823-831.

22. Merks JH, De Salvo GL, Bergeron C, Bisogno G, De Paoli A, Ferrari A, Rey A, Oberlin O, Stevens MC, Kelsey $\mathrm{A}$ et al: Parameningeal rhabdomyosarcoma in pediatric age: results of a pooled analysis from North American and European cooperative groups. Ann Oncol 2014, 25(1):231-236.

23. Spalding AC, Hawkins DS, Donaldson SS, Anderson JR, Lyden E, Laurie F, Wolden SL, Arndt CA, Michalski JM: The effect of radiation timing on patients with high-risk features of parameningeal rhabdomyosarcoma: an analysis of IRS-IV and D9803. Int J Radiat Oncol Biol Phys 2013, 87(3):512516.

24. Lucas JT, Jr., Pappo AS, Wu J, Indelicato DJ, Krasin MJ: Excessive Treatment Failures in Patients With Parameningeal Rhabdomyosarcoma With Reduced-dose Cyclophosphamide and Delayed Radiotherapy. J Pediatr Hematol Oncol 2018, 40(5):387-390.

25. Mattke AC, Bailey EJ, Schuck A, Dantonello T, Leuschner I, Klingebiel T, Treuner J, Koscielniak E: Does the time-point of relapse influence outcome in pediatric habdomyosarcomas? Pediatr Blood Cancer 2009, 52(7):772-776.

26. Dantonello TM, Int-Veen C, Winkler P, Leuschner I, Schuck A, Schmidt BF, Lochbuehler H, Kirsch S, Hallmen $E$, Veit-Friedrich I et al: Initial patient characteristics can predict pattern and risk of relapse in localized rhabdomyosarcoma. J Clin Oncol 2008, 26(3):406-413.

27. Mazzoleni S, Bisogno G, Garaventa A, Cecchetto G, Ferrari A, Sotti G, Donfrancesco A, Madon E, Casula L, Carli $\mathrm{M}$ et al: Outcomes and prognostic factors after recurrence in children and adolescents with nonmetastatic rhabdomyosarcoma. Cancer 2005, 104(1):183-190.

28. Chisholm JC, Marandet J, Rey A, Scopinaro M, de Toledo JS, Merks JH, O'Meara A, Stevens MC, Oberlin O: Prognostic factors after relapse in nonmetastatic rhabdomyosarcoma: a nomogram to better define patients who can be salvaged with further therapy. J Clin Oncol 2011, 29(10):13191325.

29. Wolden SL, Lyden ER, Arndt CA, Hawkins DS, Anderson JR, Rodeberg DA, Morris CD, Donaldson SS: Local Control for Intermediate-Risk Rhabdomyosarcoma: Results From D9803 According to 
Histology, Group, Site, and Size: A Report From the Children's Oncology Group. Int J Radiat Oncol Biol Phys 2015, 93(5):1071-1076.

30. Gallego S, Zanetti I, Orbach D, Ranchere D, Shipley J, Zin A, Bergeron C, de Salvo GL, Chisholm J, Ferrari A et al: Fusion status in patients with lymph node-positive (N1) alveolar rhabdomyosarcoma is a powerful predictor of prognosis: Experience of the European Paediatric Soft Tissue Sarcoma Study Group (EpSSG). Cancer 2018, 124(15):3201-3209.

\section{Tables}

Table 1 Follow up time of different groups.

\begin{tabular}{|llll|}
\hline & \multirow{2}{*}{ No. of cases } & \multicolumn{2}{l|}{ Follow up time (Months) } \\
\cline { 3 - 4 } & & Median & Range (min-max) \\
\hline All patients & 56 & 31.8 & $3.5-74.6$ \\
\hline Group NPD & 42 & 36.5 & $3.5-74.6$ \\
\hline Group NE & 30 & 35.6 & $3.5-73.5$ \\
\hline Group RD & 12 & 36.8 & $22.2-74.6$ \\
\hline Group PD & 14 & 17.3 & $4.8-66.0$ \\
\hline
\end{tabular}

Table 2. Clinical characteristic comparisons between Group NE, Group RD, and Group PD 


\begin{tabular}{|c|c|c|c|c|c|}
\hline & $\begin{array}{l}\text { Total } \\
\text { n (\%) }\end{array}$ & Group NE & Group RD & Group PD & $\mathrm{p}$ value \\
\hline Total cases & $56(100 \%)$ & 30 & 12 & 14 & - \\
\hline \multicolumn{6}{|l|}{ Age at diagnosis } \\
\hline$\leq 1$ y or $\geq 10$ ys & $17(30.4 \%)$ & 9 & 4 & 4 & \\
\hline Q1-9 ys & $39(69.6 \%)$ & 21 & 8 & 10 & $1.000 *$ \\
\hline \multicolumn{6}{|l|}{ Site of origin } \\
\hline Parameningeal & $32(57.1 \%)$ & 18 & 4 & 10 & \\
\hline Non-parameningeal & $24(42.9 \%)$ & 12 & 8 & 4 & $0.132 \#$ \\
\hline \multicolumn{6}{|l|}{ Tumor size } \\
\hline$\leq 5 \mathrm{~cm}$ & $37(66.1 \%)$ & 22 & 11 & 4 & \\
\hline$\otimes 5 \mathrm{~cm}$ & $19(33.9 \%)$ & 8 & 1 & 10 & $0.002 * \Delta$ \\
\hline \multicolumn{6}{|l|}{ Histologic subtype } \\
\hline Embryonal & $27(48.2 \%)$ & 16 & 8 & 3 & \\
\hline Alveolar & $29(51.8 \%)$ & 14 & 4 & 11 & $0.050 \#$ \\
\hline \multicolumn{6}{|c|}{ Primary tumor invasiveness } \\
\hline T1 & $20(35.7 \%)$ & 11 & 7 & 2 & \\
\hline $\mathrm{T} 2$ & $36(64.3 \%)$ & 19 & 5 & 12 & $0.073^{*}$ \\
\hline \multicolumn{6}{|c|}{ Regional nodal involvement } \\
\hline NO & $30(53.6 \%)$ & 17 & 8 & 5 & \\
\hline N1 & $26(46.4 \%)$ & 13 & 4 & 9 & $0.254 \#$ \\
\hline \multicolumn{6}{|l|}{ Metastasis } \\
\hline MO & $49(87.5 \%)$ & 27 & 12 & 10 & \\
\hline M1 & $7(12.5 \%)$ & 3 & 0 & 4 & $0.086 *$ \\
\hline \multicolumn{6}{|c|}{ TNM pretreatment stage } \\
\hline Stage 1 & $24(42.9 \%)$ & 12 & 8 & 4 & \\
\hline Stage 2 & $7(12.5 \%)$ & 5 & 0 & 2 & \\
\hline Stage 3 & $18(32.1 \%)$ & 10 & 4 & 4 & \\
\hline Stage 4 & $7(12.5 \%)$ & 3 & 0 & 4 & $0.119 \$$ \\
\hline
\end{tabular}




\begin{tabular}{|c|c|c|c|c|c|}
\hline Group $\otimes$ & $0(0.0 \%)$ & 0 & 0 & 0 & \\
\hline 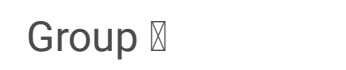 & $8(14.3 \%)$ & 5 & 3 & 0 & \\
\hline 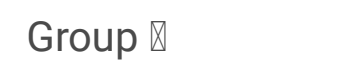 & $41(73.2 \%)$ & 22 & 9 & 10 & \\
\hline Group $\otimes$ & $7(12.5 \%)$ & 3 & 0 & 4 & $0.025 \$ \Delta$ \\
\hline \multicolumn{6}{|l|}{ Risk group } \\
\hline Low risk & $11(19.6 \%)$ & 5 & 5 & 0 & \\
\hline Intermediate risk & 38 (67.9\%) & 22 & 6 & 10 & \\
\hline High risk & $7(12.5 \%)$ & 3 & 0 & 4 & $0.002 \$ \triangle$ \\
\hline \multicolumn{6}{|l|}{ Initial RT } \\
\hline Yes & $34(60.7 \%)$ & 23 & 8 & 3 & \\
\hline No & $22(39.3 \%)$ & 7 & 4 & 11 & $0.001 * \Delta$ \\
\hline
\end{tabular}

*Fisher's exact test. \# Chi-square test. \$ Kruskal-Wallis $\mathrm{H}$ test.

A Pairwise comparisons (Bonferroni adjustment: $p<0.0167$ is considered statistically significant):

Tumor size: statistical difference between NE and PD ( $p=0.008), R D$ and PD $(p=0.002)$, no difference between NE and RD ( $p=0.247)$.

Surgical-pathologic group: statistical difference between RD and PD ( $\mathbf{p}=\mathbf{0 . 0 0 9})$, no difference between NE and PD ( $p=0.037)$, NE and RD ( $p=0.304)$.

Risk Group: statistical difference between RD and PD ( $\mathbf{p}<0.001)$, no difference between NE and PD $(p=0.057), N E$ and RD $(p=0.023)$.

Initial RT: statistical difference between NE and PD ( $\mathbf{p}=\mathbf{0 . 0 0 1})$, no difference between RD and PD $(p=0.045)$, NE and RD ( $p=0.699)$.

Table 3. Univariate comparison of risk factors between Group NPD and Group PD 


\begin{tabular}{|c|c|c|c|c|}
\hline & $\begin{array}{l}\text { Total } \\
\text { n (\%) }\end{array}$ & Group NPD & Group PD & $\mathrm{p}$ value \\
\hline Total cases & $56(100 \%)$ & 42 & 14 & - \\
\hline \multicolumn{5}{|l|}{ Age at diagnosis } \\
\hline$\leq 1$ y or $\geq 10$ ys & $39(69.6 \%)$ & 29 & 10 & \\
\hline Q1-9 ys & $17(30.4 \%)$ & 13 & 4 & $1.000 *$ \\
\hline \multicolumn{5}{|l|}{ Site of origin } \\
\hline Parameningeal & $32(57.1 \%)$ & 22 & 10 & \\
\hline Non-parameningeal & $24(42.9 \%)$ & 20 & 4 & $0.212 \#$ \\
\hline \multicolumn{5}{|l|}{ Tumor size } \\
\hline$\leq 5 \mathrm{~cm}$ & $37(66.1 \%)$ & 33 & 4 & \\
\hline$\nabla 5 \mathrm{~cm}$ & 19 (33.9\%) & 9 & 10 & $0.001 *$ \\
\hline \multicolumn{5}{|l|}{ Histologic subtype } \\
\hline Embryonal & $27(48.2 \%)$ & 24 & 3 & \\
\hline Alveolar & $29(51.8 \%)$ & 18 & 11 & $0.021 \#$ \\
\hline \multicolumn{5}{|c|}{ Primary tumor invasiveness } \\
\hline $\mathrm{T} 1$ & $20(35.1 \%)$ & 18 & 2 & \\
\hline T2 & $36(64.3 \%)$ & 24 & 12 & $0.053 \#$ \\
\hline \multicolumn{5}{|c|}{ Regional nodal involvement } \\
\hline NO & $30(53.6 \%)$ & 25 & 5 & \\
\hline N1 & $26(46.4 \%)$ & 17 & 9 & $0.122 \#$ \\
\hline \multicolumn{5}{|l|}{ Metastasis } \\
\hline MO & 49 (87.5\%) & 39 & 10 & \\
\hline M1 & $7(12.5 \%)$ & 3 & 4 & $0.058 *$ \\
\hline \multicolumn{5}{|c|}{ TNM pretreatment stage } \\
\hline Stage 1 & $24(42.9 \%)$ & 20 & 4 & \\
\hline Stage 2 & $7(12.5 \%)$ & 5 & 2 & \\
\hline Stage 3 & $18(32.1 \%)$ & 14 & 4 & \\
\hline Stage 4 & $7(12.5 \%)$ & 3 & 4 & $0.095 \$$ \\
\hline
\end{tabular}




\begin{tabular}{|c|c|c|c|c|}
\hline Group $\otimes$ & $0(0.0 \%)$ & 0 & 0 & \\
\hline Group $\otimes$ & $8(19.0 \%)$ & 8 & 0 & \\
\hline Group $\nabla$ & 31 (73.8\%) & 31 & 10 & \\
\hline 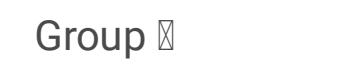 & $3(7.1 \%)$ & 3 & 4 & $0.011 \$$ \\
\hline \multicolumn{5}{|l|}{ Risk group } \\
\hline Low risk & $11(19.6 \%)$ & 11 & 0 & \\
\hline Intermediate risk & 38 (67.9\%) & 28 & 10 & \\
\hline High risk & $7(12.5 \%)$ & 3 & 4 & $0.006 \$$ \\
\hline \multicolumn{5}{|l|}{ Initial RT } \\
\hline Yes & $34(60.7 \%)$ & 31 & 3 & \\
\hline No & 22 (39.3\%) & 11 & 11 & $0.001 \#$ \\
\hline
\end{tabular}

* Fisher's exact test. \# Chi-square test. \$ Cochran-Armitage trend test.

Table 4. Multivariate analysis of results of binary logistic model.

\begin{tabular}{|llll|}
\hline Risk Factors & \multicolumn{3}{l|}{ Disease Progression } \\
\cline { 2 - 4 } & OR & $95 \%$ Cls & $\mathrm{p}$ \\
\hline Tumor size $>5 \mathrm{~cm}$ & 6.75 & $1.02-44.78$ & $\mathbf{0 . 0 4 8}$ \\
\hline Alveolar histology & 7.10 & $1.08-46.57$ & $\mathbf{0 . 0 4 1}$ \\
\hline Non-initial RT & 15.26 & $2.38-97.70$ & $\mathbf{0 . 0 0 4}$ \\
\hline Higher ranks of surgical-pathologic group & 1.54 & $0.24-9.77$ & 0.646 \\
\hline
\end{tabular}

\section{Figures}




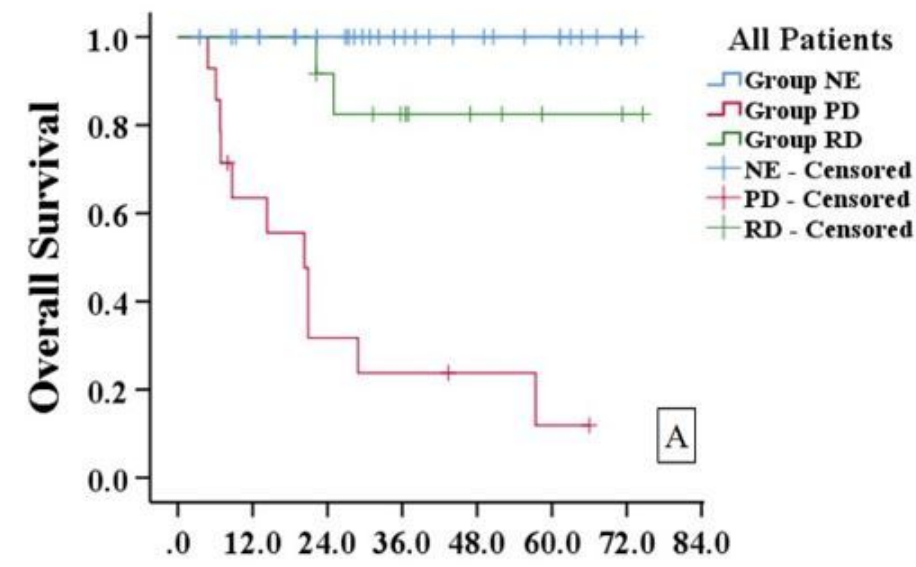

Survival Time (Months)

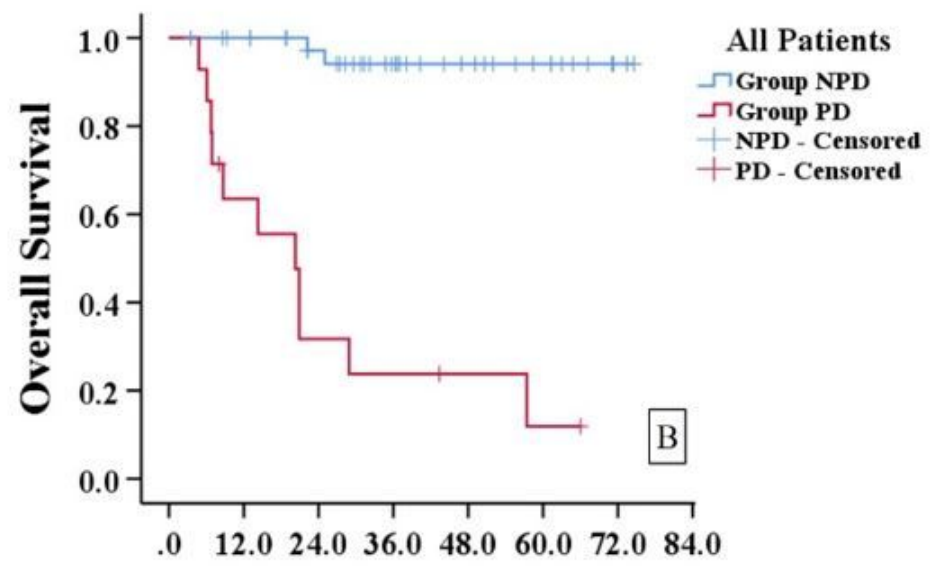

Survival Time (Months)

\section{Figure 1}

Kaplan-Meier survival curve. A. OS of Group NE, Group RD, and Group PD $(p<0.001)$; pairwise comparisons with Bonferroni adjustment showed statistical differences between Group NE and Group PD $(p<0.001)$, as well as Group PD and Group RD $(p=0.001)$, but no difference between Group NE and Group RD ( $p=0.043)$. B. OS of Group NPD and Group PD $(p<0.001)$. 